07

\title{
Арсенид-галлиевые детекторы с барьером Шоттки для спектрометрии альфа-частиц при температурах до $120^{\circ} \mathrm{C}$
}

\author{
(C) А.В. Черных ${ }^{1}$, С.В. Черных ${ }^{1}$, С.И. Диденко ${ }^{1}$, Н. Буртебаев ${ }^{2}$, \\ Маулен Насурлла ${ }^{2,3}$, Маржан Насурлла ${ }^{2,3}$, Г.И. Бритвич ${ }^{4}$, \\ А.П. Чубенко ${ }^{5}$ Ф.М. Барышников ${ }^{1}$, Е.В. Слепцов ${ }^{1}$
}

\author{
${ }^{1}$ Национальный исследовательский технологический университет \\ „МИСиС“, Москва, Россия \\ ${ }^{2}$ Институт ядерной фризики Министерства энергетики Республики \\ Казахстан, Алматы, Казахстан \\ ${ }^{3}$ Казахский национальный университет им. аль-Фараби, Алматы, \\ Казахстан \\ ${ }^{4}$ Институт фризики высоких энергий им. А.А. Логунова НИЦ „Курчатовский \\ институт“, Протвино, Московская обл., Россия \\ ${ }_{5}^{5}$ Физический институт им. П.Н. Лебедева РАН, Москва, Россия \\ E-mail: chav_84@mail.ru
}

Поступило в Редакцию 21 ноября 2017 г.

Сообщается о результатах испытаний арсенид-галлиевых детекторов для спектрометрии $\alpha$-частиц. Детекторы имели площадь входного окна $80 \mathrm{~mm}^{2}$ и толщину рабочего слоя 40-50 $\mu \mathrm{m}$. Энергетическое разрешение FWHM, измеренное на $\alpha$-линии $5.499 \mathrm{MeV}$ источника ${ }^{238} \mathrm{Pu}$, составило $17.5 \mathrm{keV}$ при ширине пика генератора $7.8 \mathrm{keV}$. При измерениях на источнике ${ }^{226} \mathrm{Ra}$ детекторы демонстрировали линейный отклик и эффективность сбора заряда, близкую к $100 \%$, при обратном смещении более $65 \mathrm{~V}$ для всех энергий $\alpha$-частиц, испускаемых источником. Проведенные испытания на температурную устойчивость показали возможность использования разработанных детекторов для $\alpha$-спектрометрии радионуклидов при температурах до $120^{\circ} \mathrm{C}$.

DOI: $10.21883 /$ PJTF.2018.20.46810.17130

В настоящее время бо́льшая часть полупроводниковых детекторов изготавливается из кремния. Это обусловлено высокими детекторными параметрами материала и его высочайшей технологичностью, что позволяет создавать детекторы различных классов и конструкций [1]. Однако кремниевые детекторы не способны удовлетворить требованиям 
части современных ускорительных и ядерно-физических экспериментов, а также некоторых других приложений. Для их замены в случае жестких условий эксплуатации целесообразна разработка ядерных детекторов на основе широкозонных материалов ( $\mathrm{GaN}, \mathrm{SiC}$, алмаз, GaAs и др.) вследствие большей радиационной и температурной устойчивости последних.

Характеристики детекторов на основе $\mathrm{GaN}$ пока неудовлетворительны [2], несмотря на то что технология приборов различных классов на базе этого материала уже достаточно развита. Их улучшение напрямую связано с повышением качества материала: снижением плотности дислокаций, концентрации ловушек и фонового легирования. Первые обнадеживающие результаты для $\mathrm{GaN}$ достигнуты совсем недавно: полученное в [3] разрешение FWHM (full width at half maximum) составило $2.2 \%(121 \mathrm{keV})$ на линии $5.486 \mathrm{MeV}$ при смещении $-550 \mathrm{~V}$ и площади детектора $0.8 \mathrm{~mm}^{2}$. Детекторы на основе алмаза и карбида кремния, напротив, имеют приемлемые характеристики и активно тестируются для использования в экспериментах. Так, например, детекторы частиц спектрометрического качества на алмазе (FWHM $0.3-0.4 \%$ для $\alpha$-частиц с энергией $5.5 \mathrm{MeV}$ ) площадью до $16 \mathrm{~mm}^{2}$ уже представлены $[4,5]$. Приборы на основе $\mathrm{SiC}$ также демонстрируют характеристики, близкие к таковым для кремниевых детекторов: в [6] получено разрешение FWHM $16.2 \mathrm{keV}$ с использованием детектора площадью $11 \mathrm{~mm}^{2}$, в [7] сообщается о получении FWHM $13.8 \mathrm{keV}$ на линии $5.486 \mathrm{MeV}^{241} \mathrm{Am}$ (площадь детектора $0.6 \mathrm{~mm}^{2}$ ). Отметим, что, несмотря на полученные результаты, высокая стоимость и относительно небольшие достигнутые площади детекторов на основе $\mathrm{SiC}$ и алмаза пока не позволяют говорить о возможности их широкого применения. В связи с этим для большинства приложений при температурах до $100^{\circ} \mathrm{C}$, требующих достаточно высокой радиационной стойкости (в сравнении с кремнием), более дешевым решением может быть использование детекторов на основе GaAs.

B [8] сообщается о разработке детекторов на основе VPE-GaAs (VPE - vapor-phase epitaxy) с тонким барьером Шоттки площадью до $80 \mathrm{~mm}^{2}$. Лучшее измеренное энергетическое разрешение FWHM составило $14.2 \mathrm{keV}$ при площади детектора $25 \mathrm{~mm}^{2}$, что значительно превышает параметры представленных ранее детекторов на основе GaAs. Настоящая работа является продолжением этого исследования и посвящена испытаниям поверхностно-барьерных детекторов на базе

Письма в ЖТФ, 2018, том 44, вып. 20 


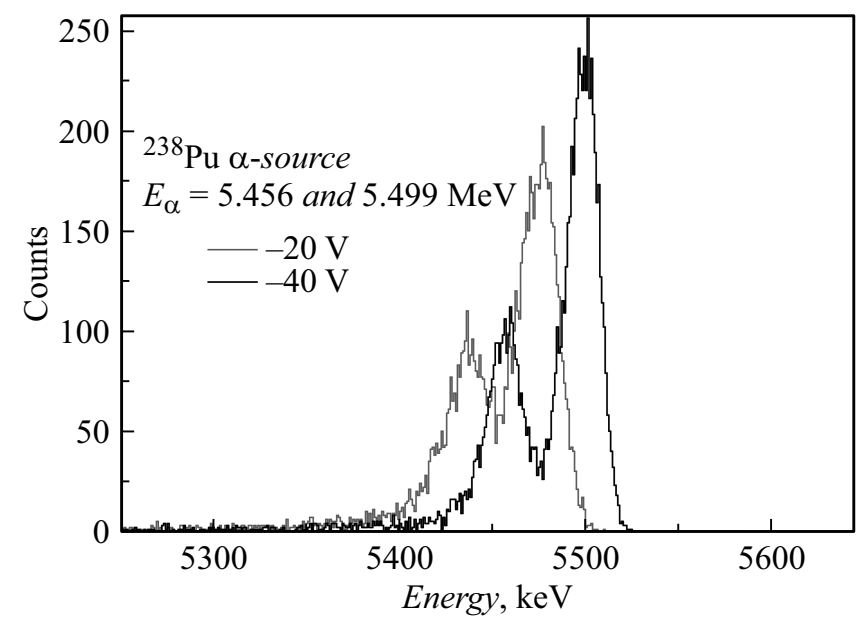

Рис. 1. Энергетический спектр $\alpha$-частиц от источника ${ }^{238} \mathrm{Pu}$ при смещениях -20 и $-40 \mathrm{~V}$.

эпитаксиального GaAs при облучении $\alpha$-частицами при температурах выше комнатной.

Экспериментальные образцы детекторов изготавливались на основе высокоомных эпитаксиальных слоев VPE-GaAs толщиной 40-50 $\mu \mathrm{m}$. В качестве выпрямляющего контакта использовался барьер Шоттки площадью $80 \mathrm{~mm}^{2}$ на основе системы металлизации Pt/TiN суммарной толщиной $0.11 \mu \mathrm{m}$, в качестве омического контакта к $n^{++}$-подложке система $\mathrm{Ni} / \mathrm{AuGe} / \mathrm{Au}$. Изоляция активной области осуществлялась посредством формирования меза-структуры с последующей пассивацией полиимидом. Конструкция и электрические характеристики детекторов детально представлены в [9].

Оценка спектрометрического качества детекторов проводилась аналогично [8]. На рис. 1 представлены типичные спектры $\alpha$-частиц от источника ${ }^{238} \mathrm{Pu}$, полученные при различных напряжениях смещения на детекторе. Можно видеть, что обе линии источника хорошо разрешаются уже при смещении $-20 \mathrm{~V}$, при этом FWHM для линии $5.499 \mathrm{MeV}$ составляет $23.0 \mathrm{keV}$. С дальнейшим увеличением смещения по абсолютной величине до $-40 \mathrm{~V}$ энергетическое разрешение улучшается до $17.5 \mathrm{keV}$ при энергетическом эквиваленте шума $7.8 \mathrm{keV}$.

Письма в ЖТФ, 2018, том 44, вып. 20 


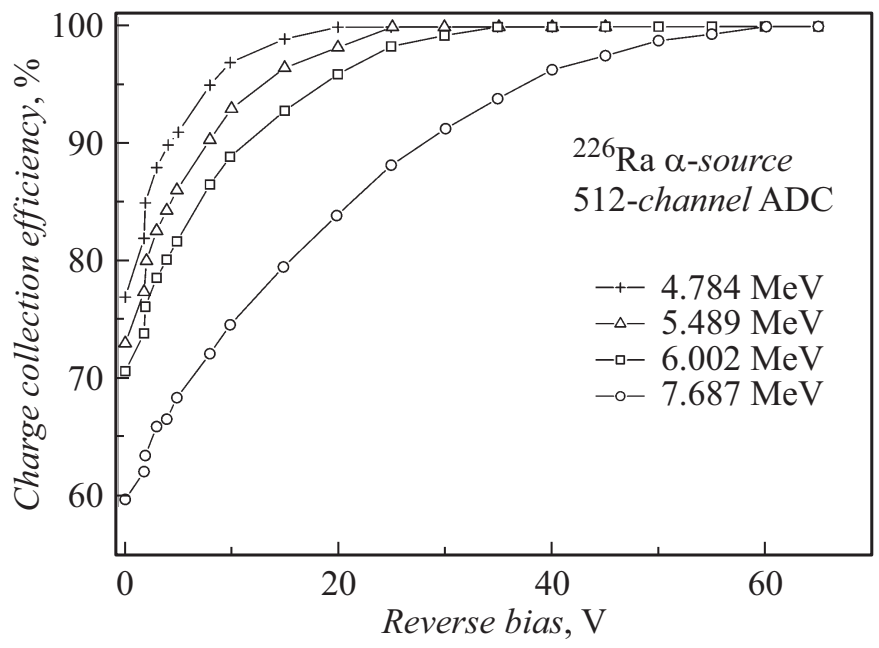

Рис. 2. Типичная зависимость эффективности сбора заряда от рабочего смещения для исследуемых детекторов на VPE-GaAs, измеренная для различных $\alpha$-линий ${ }^{226} \mathrm{Ra}$.

На рис. 2 представлено характерное поведение зависимости эффективности сбора заряда (CCE - charge collection efficiency) разработанных детекторов от приложенного смещения для $\alpha$-частиц от источника ${ }^{226} \mathrm{Ra}$. Видно, что ССЕ от самых длиннопробежных $\alpha$-частиц выходит на максимум при значительно бо́льших обратных смещениях $($ не менее $70 \mathrm{~V})$. Это можно объяснить тем, что с увеличением пробега $\alpha$-частиц, согласно теореме Рамо-Шокли [10], возрастает вклад дырочной составляющей в наведенный сигнал (и соответственно ССЕ) и снижается вклад электронной, поскольку область максимальных ионизационных потерь на кривой Брэгга смещается в сторону положительного электрода. Отметим, что расчет пробегов в Geant 4 для $\alpha$-частиц с энергиями $4.784,5.489,6.002$ и $7.687 \mathrm{MeV}$ в $\mathrm{GaAs}$ дает значения $18,21.5,24$ и $34 \mu \mathrm{m}$. Такое различие пробегов $\alpha$ частиц источника в сочетании со значительными рекомбинационными потерями заряда в плазменном треке при низких смещениях может иметь сильное влияние на вид зависимости ССЕ от энергии для исследуемых детекторов. 
Измерения температурных характеристик проводились в вакууме при остаточном давлении $100 \mathrm{~Pa}$. Источник питания, мультиметр и предусилитель были вынесены за пределы вакуумной камеры и соединялись соответственно с нагревателем (резистивного типа), термопарой и детектором посредством вакуумных разъемов. Для поддержания постоянной температуры детектора установка была оснащена системой автоматического регулирования тока. При каждой температуре контролировался обратный ток при напряжении смещения $-100 \mathrm{~V}$. При комнатной температуре он определялся утечкой и составил $1.5 \mathrm{nA}$, при температуре более $100^{\circ} \mathrm{C}$ преобладающей становилась генерационная составляющая, и при $130^{\circ} \mathrm{C}$ обратный ток составлял $3 \mu \mathrm{A}$.

Результаты измерений энергетических спектров $\alpha$-частиц от источника ${ }^{226} \mathrm{Ra}$ для различных температур и приложенных смещений представлены на рис. 3. Измеренное при комнатной температуре для всех линий энергетическое разрешение (FWHM) практически не различалось и составило в среднем $50.8 \mathrm{keV}$ (напряжение смещения $-60 \mathrm{~V})$. Отметим, что в данном случае разрешение определялось флуктуацией потерь энергии $\alpha$-частиц в защитном покрытии источника $\mathrm{FWHM}_{\text {sourse }} \approx 36 \mathrm{keV}$ и шумами электроники $\mathrm{FWHM}_{\text {gen }} \approx 27 \mathrm{keV}$. При увеличении температуры до $60^{\circ} \mathrm{C}$ спектральные характеристики детектора оставались практически неизменными. С дальнейшим увеличением температуры до $80^{\circ} \mathrm{C}$ (рис. 3) энергетическое разрешение для низкоэнергетических пиков незначительно ухудшилось $(51.8 \mathrm{keV})$ за счет увеличения шумов $\left(\mathrm{FWHM}_{\text {gen }}=34.4 \mathrm{keV}\right)$, однако для линии $7.687 \mathrm{MeV}$ изменение было более заметным $(67.7 \mathrm{keV})$. При $100^{\circ} \mathrm{C}$ для пика $7.687 \mathrm{MeV}$ наблюдался значительный дефицит амплитуды (около $250 \mathrm{keV}$ ) и серьезно ухудшилось энергетическое разрешение до $266 \mathrm{keV}$. Для других линий энергетическое разрешение также снизилось, при этом его значение согласовывалось с энергиями пиков (4.784 MeV - 61.4 keV, $5.489 \mathrm{MeV}-66.5 \mathrm{keV}, 6.002 \mathrm{MeV}-75.0 \mathrm{keV}$ при шуме $48.7 \mathrm{keV}$ ). Подобное поведение спектра с температурой следует связывать с особенностями сбора заряда в детекторах с тонким рабочим слоем, как было показано выше, и с уменьшением параметров переноса $\mu \tau$ из-за снижения дрейфовой подвижности носителей заряда $\mu$ вследствие фононного рассеяния, а также снижения времени жизни $\tau$, обусловленного увеличением концентрации ионизированных центров рекомбинации (прежде всего центов EL2). Увеличение обратного смещения до $90 \mathrm{~V}$ позволило нивелировать

Письма в ЖТФ, 2018, том 44, вып. 20 

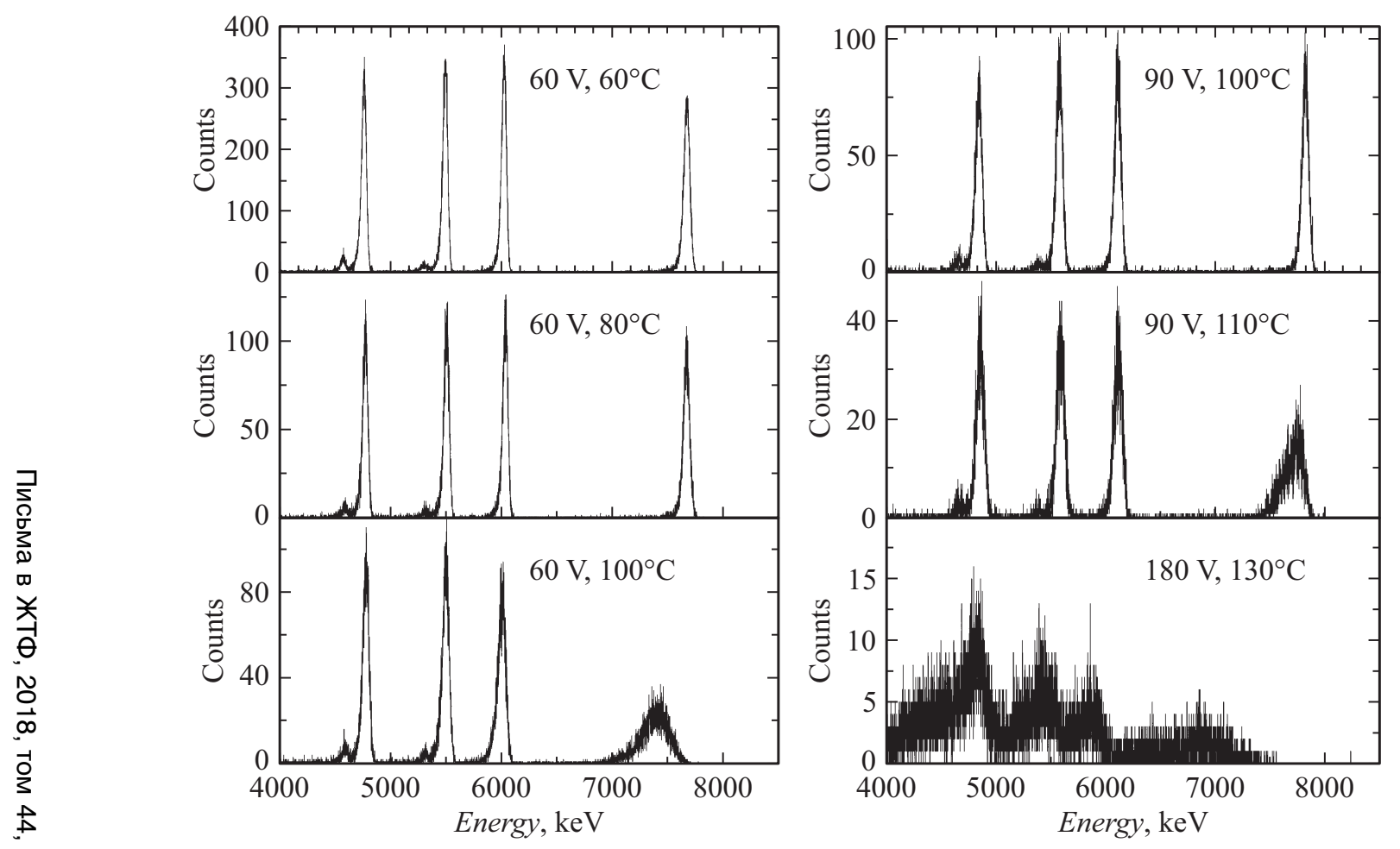

Рис. 3. Энергетический спектр $\alpha$-частиц от источника ${ }^{226} \mathrm{Ra}$, измеренный с помощью детектора на VPE-GaAs при различных температурах и напряжениях смещения. 
влияние температуры (рис. 3), и, как следствие, характеристики детектора были близки к таковым при комнатной температуре. Однако по причине более высоких шумов детектора $\left(\mathrm{FWHM}_{\text {gen }}=55.5 \mathrm{keV}\right)$, связанных с ростом генерационного тока, энергетическое разрешение детектора было несколько хуже $-69.2 \mathrm{keV}$ (независимо от энергии пика). Как показал дальнейший эксперимент, детектор полностью сохранял спектрометрические качества до температуры порядка $120^{\circ} \mathrm{C}$ (для всех линий FWHM находилось на уровне $135 \mathrm{keV}$ ), однако при этом было необходимо увеличивать рабочее смещение до 180-190 V. Увеличение температуры более $130^{\circ} \mathrm{C}$ приводило к катастрофическому ухудшению характеристик: детектор не „различал“ отдельные линии источника. Отметим, что при $130^{\circ} \mathrm{C}$, несмотря на сильное снижение ССЕ, детектор все еще сохранял работоспособность (рис. 3).

В дальнейшем поведение спектральных характеристик разработанных детекторов в зависимости от температуры будет детально изучено и обсуждено. Однако уже сейчас можно утверждать, что их температурный диапазон работы ограничивается $120^{\circ} \mathrm{C}$, что в первую очередь обусловлено наличием в материале глубокого центра EL2. Оценочные измерения методом нестационарной емкостной спектроскопии глубоких уровней дали значение концентрации этого центра на уровне $(1-5) \cdot 10^{13} \mathrm{~cm}^{-3}$. Можно утверждать, что полученный результат значительно превосходит характеристики кремниевых детекторов, а также детекторов на основе SI LEC-GaAs, которые теряют свои спектрометрические качества уже при температуре $50^{\circ} \mathrm{C}$ [11]. Необходимо также добавить, что, возможно, это предел для GaAs, поскольку эпитаксиальные слои, выращиваемые с помощью газофазной эпитаксии, являются более „чистыми“ по сравнению с объемными полуизолирующими материалами, в которых концентрация центров EL2 находится на уровне $10^{15} \mathrm{~cm}^{-3}$.

Таким образом, проведенные температурные испытания показали возможность использования детекторов на основе $\mathrm{GaAs}$ в спектрометрическом режиме до температур $120^{\circ} \mathrm{C}$. Полученный результат позволяет надеяться на то, что изготовленные приборы могут быть успешно использованы для спектрометрии радионуклидов в условиях производства атомной промышленности, например для контроля остаточной радиоактивности сборок с отработавшим ядерным топливом.

Письма в ЖТФ, 2018, том 44, вып. 20 
Авторы благодарят Ю.Н. Глыбина (СНИИП-Плюс, Москва) за проведенные испытания детекторов при оценке их энергетического разрешения и обсуждение результатов.

Работа выполнена при финансовой поддержке Министерства образования и науки РФ в рамках государственного задания вузу № 3.2794.2017/4.6.

\section{Список литературы}

[1] Hartmann F. Evolution of silicon sensor technology in particle physics. Springer Int. Publ., 2017. V. 275. N 2. 372 p.

[2] Wang J., Mulligan P., Brillson L., Cao L.R. // Appl. Phys. Rev. 2015. V. 2 . N 3. P. 031102.

[3] Xu Q., Mulligan P., Wang J., Chuirazzi W., Cao L. // Nucl. Instrum. Meth. Phys. Res. A. 2017. V. 849. P. 11-15.

[4] Pomorski M., Berdermann E., Caragheorgheopol A., Ciobanu M., Kiš M., Martemiyanov A., Nebel C., Moritz P. // Phys. Status Solidi A. 2006. V. 203. N 12. P. $3152-3160$.

[5] Dueñas J.A., de la Torre Pérez J., Sánchez A.M., Martel I. // Appl. Rad. Isotop. 2014. V. 90. P. $177-180$.

[6] Chaudhuri S.K., Zavalla K.J., Mandal K.C. // Nucl. Instrum. Meth. Phys. Res. A. 2013. V. 728. P. 97-101.

[7] Zat'ko B., Dubeckýa F., Šagátováb A., Sedlačováb K., Ryćc L. // J. Instrum. 2015. V. 10. P. C04009.

[8] Chernykh S.V., Chernykh A.V., Didenko S.I., Baryshnikov F.M., Burtebayev N., Britvich G.I., Chubenko A.P., Guly V.G., Glybin Yu.N., Zholdybayev T.K., Burtebayeva J.T., Nassurlla M. // Nucl. Instrum. Meth. Phys. Res. A. 2017. V. 845. P. 52-55.

[9] Chernykh A.V., Chernykh S.V., Baryshnikov F.M., Didenko S.I., Burtebayev N., Britvich G.I., Kostin M.Yu., Chubenko A.P., Nassurlla Marzhan, Nassurlla Maulen // J. Instrum. 2016. V. 11. N 12. P. C12005.

[10] Ramo S. // Proc. of the IRE. 1939. V. 27. N 9. P. 584-585.

[11] Беспалов В.А., Воронцов А.В., Горбацевич А.А., Егоркин В.И., Жигальский Г.П., Ильичев Э.А., Кулаков А.В., Налбандов Б.Г., Пантуев В.С., Распутный В.Н., Свешников Ю.Н., Шмелев С.С. // ЖТФ. 2004. Т. 74. В. 3. C. 28-36. 